\title{
Using Graphene Liquid Cells for High-resolution Chemical Analysis of Nano- particle Reactions
}

\author{
Canhui Wang ${ }^{1}$, Tolou Shokuhfar ${ }^{1,2}$ and Robert F Klie ${ }^{1}$ \\ 1. University of Illinois at Chicago, Department of Physics, Chicago, IL 60607 \\ 2. Michigan Technological University, Department of Mechanical Engineering, Dept. of Biomedical \\ Engineering, Houghton, MI, USA 49931
}

Radiolysis has been intensely studied due to its importance in areas, such as medicine, atmospheric science and nuclear energy production. In transmission electron microscopy, however, where the dose rate of a $200 \mathrm{keV}$ electron beam is 7 orders of magnitude greater that dose rates commonly generated by other radiation sources, radiolysis has mostly been discarded as an unwanted side-effect. For in-situ TEM experiments, the creation of unwanted free radicals, such as hydroxyl and hydrogen, or hydrated electrons, as the result of water dissociation by a high energy electron beam, can have a significant effects on the stability of a suspended nano-particle or on the redox conditions in an electro-chemical experiment. Only recently was it realized that a fundamental understanding and control of radiolysis is required in order to reliably study chemical reactions in an electron microscope. To date, a few experiments [1,2] and theoretical studies have been reported in the literature,[3] addressing the fundamental issues of radiolysis. Therefore, even a basic understanding of the observed effects of radiolysis is still lacking.

Direct imaging of electron-liquid interaction has become possible at the nanometer scale with the widespread availability of commercial liquid cells, where a small volume of liquid is sealed between two electron transparent layers. While this enables thin liquid layers, and structures suspended in such layers, to be studied at atmospheric pressures in processes such as electroplating, nucleation of nanoparticles or bubbles, and biological reaction, the imaging resolution and chemical quantification capabilities are severely limited by the existing holder designs. Moreover, the electron dose rate required for atomicresolution imaging is believed to be significantly higher than the damage threshold in conventional liquid-cell experiments.

We have recently developed a novel approach towards in-situ electron microscopy that allows the effects of radiolysis to be examined on the atomic-level scale and provides the required control over these effects to minimize beam-induced radiation damage. More specifically, we utilize a liquid cell, consisting of two graphene monolayers to encapsulate a small volume of liquid, as shown in Figure 1.[4] Such an approach allow us to image particles, such as the $12 \mathrm{~nm}$ diameter iron core of the protein ferritin with atomic resolution in a liquid environment. Moreover, electron energy-loss spectroscopy and spectrum imaging of the core-loss edges is now also possible for samples in a graphene liquid cell (Figure 2), since the window layers consist of only a monolayer of carbon instead of $>20 \mathrm{~nm}$ of $\mathrm{SiN}$.

In addition to the high spatial and spectra resolution that can be achieved using this new approach, we also discuss the effects of electron dose on the GLCs. We observed the generation of bubbles inside the GLC at sufficiently high electron dose rates. However, GLCs do not generate bubbles under even hours of electron beam irradiation at low magnification, indicating accumulation of total electron dose is not the reason for bubble formation in this system. This will be critical for our understanding of radiolysis 
and will be discussed in the context of the recent modeling study by Schneider et al. [3] Applications of this method to catalysts nanoparticles and battery cathode materials will be discussed. [5]

\section{References:}

[1] Grogan, J.M. et al, Nano Letters 14(1) (2014), p. 359.

[2] Zheng, H., et al, Nano Letters 9(6) (2009), p. 2460.

[3] Schneider, N.M., et al, Journal of Heat Transfer-Transactions of the Asme 136(8) (2014)

[4] Wang, C., et al, Advanced Materials 26 (2014), p. 3410.

[5] This research was supported by Michigan Technological University and Research Resource Center, University of Illinois at Chicago.

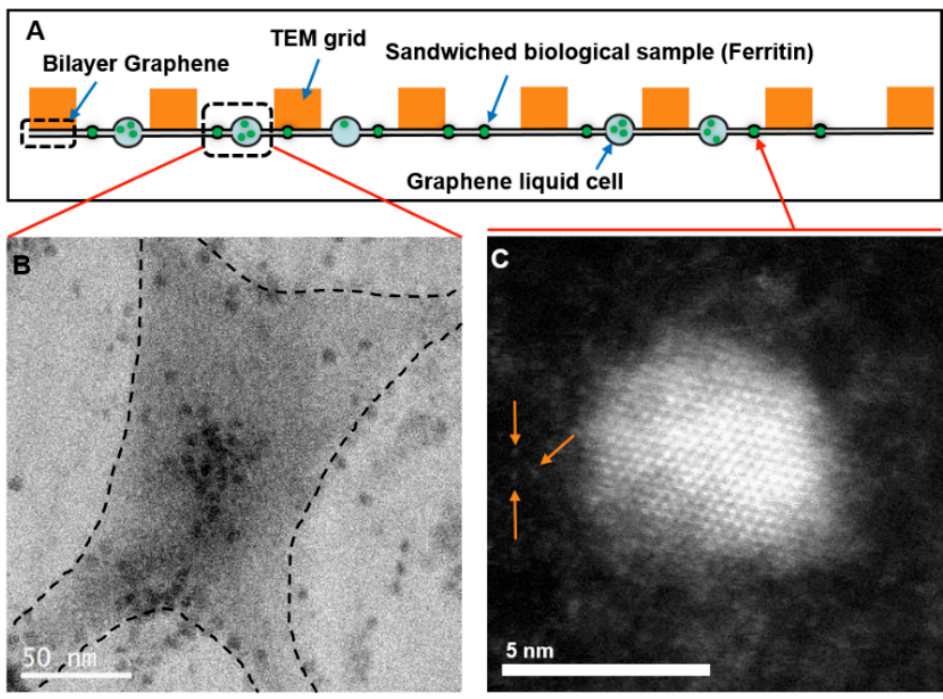

Figure 1: Schematic diagram (A), as well as STEM images (B) and (C) of ferritin molecules in GLCs and graphene sandwiches. (B) is an annular bright field (ABF) image showing ferritin molecules encapsulated in both a GLC and graphene layers. The edges of the GLC is indicated by dash lines. (C) is a high angle annular dark field (HAADF) image showing atomic-resolution image of a sandwiched ferritin molecule, with the $12 \mathrm{~nm}$ in diameter protein shell and individual $\mathrm{Fe}$ atoms resolved in light contrast.

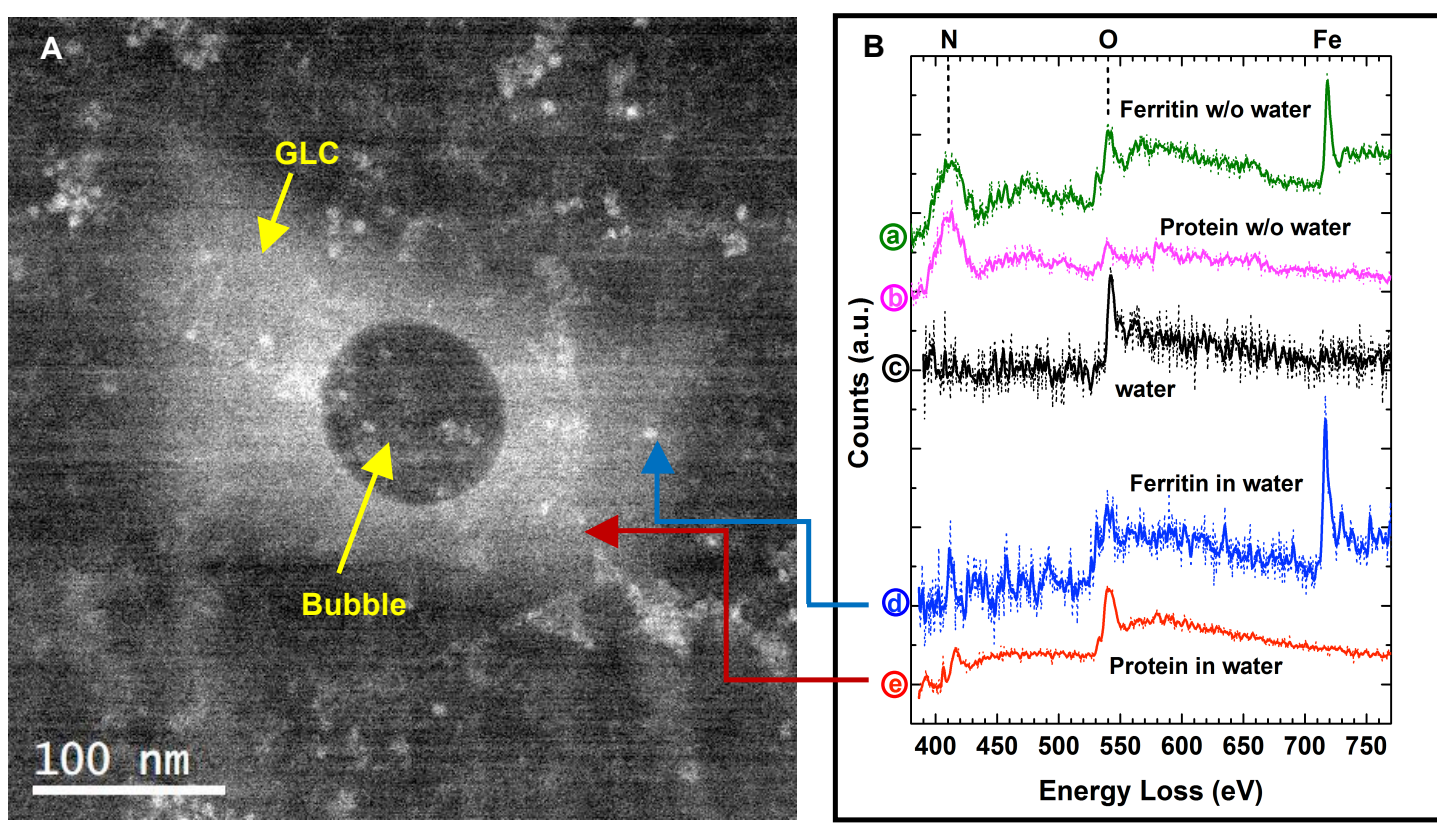

Figure 2: (A) HAADF image of GLC containing ferritin molecules as well as a gas bubble in the center. (B) EELS spectra of the $\mathrm{N}$ $K$-, $\mathrm{O} K$ - end $\mathrm{Fe}$ $L$-edges taken from ferritin core and the protein shell in water as well as from regions without water. 